Full length article

\title{
Enhanced chondrogenic phenotype of primary bovine articular chondrocytes in Fibrin-Hyaluronan hydrogel by multi-axial mechanical loading and FGF18
} \author{
Sibylle Grad ${ }^{\mathrm{c}}$, Avner Yayon ${ }^{\mathrm{a}, \mathrm{b}, *}$ \\ a Institute of Biochemistry, Food Science and Nutrition, The Hebrew University of Jerusalem, Rehovot, Israel \\ b Procore Ltd., Weizmann Science Park, 7 Golda Meir St., P.O. Box 4082, Ness Ziona 7414002, Israel \\ ${ }^{\mathrm{c}}$ AO Research Institute, Davos, Switzerland \\ ${ }^{\mathrm{d}}$ Department of Orthopedics, Erasmus MC, University Medical Center, CN Rotterdam, the Netherlands
}

Bernardo P. Antunes ${ }^{a, b}$, M. Letizia Vainieri ${ }^{c, d}$, Mauro Alini ${ }^{c}$, Efrat Monsonego-Ornan ${ }^{a}$,

\section{A R T I C L E I N F O}

\section{Article history:}

Received 16 September 2019

Revised 21 January 2020

Accepted 21 January 2020

Available online 23 January 2020

\section{Keywords:}

Chondrogenic differentiation

Fibrin-hyaluronan hydroge

Fibroblast growth factor-18

Multi-axial loading

\begin{abstract}
A B S T R A C T
Current treatments for cartilage lesions are often associated with fibrocartilage formation and donor site morbidity. Mechanical and biochemical stimuli play an important role in hyaline cartilage formation. Biocompatible scaffolds capable of transducing mechanical loads and delivering bioactive instructive factors may better support cartilage regeneration.

In this study we aimed to test the interplay between mechanical and FGF-18 mediated biochemical signals on the proliferation and differentiation of primary bovine articular chondrocytes embedded in a chondro-conductive Fibrin-Hyaluronan (FB/HA) based hydrogel.

Chondrocytes seeded in a Fibrin-HA hydrogel, with or without a chondro-inductive, FGFR3 selective FGF18 variant (FGF-18v) were loaded into a joint-mimicking bioreactor applying controlled, multi-axial movements, simulating the natural movements of articular joints. Samples were evaluated for DNA content, sulphated glycosaminoglycan (sGAG) accumulation, key chondrogenic gene expression markers and histology.

Under moderate loading, samples produced particularly significant amounts of sGAG/DNA compared to unloaded controls. Interestingly there was no significant effect of FGF-18v on cartilage gene expression at rest. Following moderate multi-axial loading, FGF-18v upregulated the expression of Aggrecan (ACAN), Cartilage Oligomeric Matrix Protein (COMP), type II collagen (COL2) and Lubricin (PRG4). Moreover, the combination of load and FGF-18v, significantly downregulated Matrix Metalloproteinase-9 (MMP-9) and Matrix Metaloproteinase-13 (MMP-13), two of the most important factors contributing to joint destruction in OA. Biomimetic mechanical signals and FGF-18 may work in concert to support hyaline cartilage regeneration and repair.
\end{abstract}

\section{Statement of significance}

Articular cartilage has very limited repair potential and focal cartilage lesions constitute a challenge for current standard clinical procedures. The aim of the present research was to explore novel procedures and constructs, based on biomaterials and biomechanical algorithms that can better mimic joints mechanical and biochemical stimulation to promote regeneration of damaged cartilage.

Using a hydrogel-based platform for chondrocyte 3D culture revealed a synergy between mechanical forces and growth factors. Exploring the mechanisms underlying this mechano-biochemical interplay may enhance our understanding of cartilage remodeling and the development of new strategies for cartilage repair and regeneration.

(C) 2020 Acta Materialia Inc. Published by Elsevier Ltd. All rights reserved.

\footnotetext{
* Corresponding author at: Procore Ltd., Weizmann Science Park, 7 Golda Meir St., P.O. Box 4082, Ness Ziona 7414002, Israel.

E-mail address: yayon@procore-bio.com (A. Yayon).
} 


\section{Introduction}

Articular cartilage is a highly specialized tissue that provides low friction and allows for efficient load bearing and distribution. The major cartilage constituents comprise a highly hydrated and organized extracellular matrix (ECM), consisting mostly of collagen fibres and proteoglycans, and a low density of specialized chondrocytes [1]. Articular cartilage is non-vascularized, non-innervated and lacks a supporting perichondrial layer, therefore, once damaged, it does not elicit effective tissue repair responses. Cartilage damage and associated catabolic processes are usually irreversible and often lead to permanent cartilage loss and osteoarthritis (OA) [2].

Different strategies over the years have attempted to regenerate cartilaginous tissue. Surgical techniques, such as abrasive chondroplasty, microfracture and spongialization, failed to achieve authentic tissue repair, but, instead, formed fibrocartilaginous tissue, which does not possess the mechanical properties of normal healthy cartilage $[3,4]$. Another procedure receiving much attention is autologous chondrocyte implantation (ACI). However, ACI usually requires multiple surgeries, along with long periods of recovery and rehabilitation. On the other hand, matrix-associated ACI (MACI), applies an exogenous matrix that can improve the mechanical stability and durability of the implanted cells as well as provide a proper stimulus for chondrogenic differentiation and cartilage regeneration $[5,6]$.

Several studies have demonstrated that fibrin-based hydrogels provide a most suitable environment for multiple cell functions, i.e. migration, proliferation and differentiation [7-9]. Chondrocytes embedded in fibrin hydrogels retain their rounded differentiated morphology and produce cartilaginous ECM $[10,11]$. However, fibrin particularly when subjected to the harsh environment of $\mathrm{OA}$, undergoes fibrinolysis and loss of scaffold stability [12]. While rapid degradation can be an advantage in some applications (e.g. wound dressing), it represents a limitation for cartilage repair. Long-term stability of the scaffold is required to provide enough time for cell proliferation, differentiation and matrix production [13].

Efforts were therefore made to add bio-macromolecules to the fibrin hydrogel to improve its stability [14]. Incorporation of hyaluronic acid (HA) into fibrin-based scaffolds decreases the fibrinolysis rate and improves the mechanical and biological properties in-vitro and in-vivo [15-17]. HA, a major component of articular cartilage and synovial fluid, supports cell proliferation and maintains the chondrogenic phenotype, increasing the production of cartilaginous ECM [18-21].

Fibrin-hyaluronan hydrogels have been described as adequate platforms for cartilage regeneration, able to crosslink in situ, at body temperature, rendering the system safely injectable and minimally invasive $[8,16,22-24]$. These have been shown to increase the secretion of extracellular matrix components, such as GAG and collagen, when compared to chondrocytes embedded in agarose or alginate gels [14]. Cell-hydrogel constructs develop increased mechanical strength following the deposition of extracellular matrix enriched in collagen type II, a hallmark of hyaline cartilage [16]. In gel matrix deposition may facilitate the conductance of intraarticular mechanical stimuli which have been shown to be of critical importance in stimulating the development of normal articular hyaline cartilage [16].

Various anabolic compounds have been evaluated to promote cartilage regeneration $[25,26]$. In mature articular chondrocytes, fibroblast growth factor-18 (FGF-18) exhibits mitogenic activities in addition to increased ECM production, thereby promoting cartilage repair, in both in vitro and in vivo models [27-31]. N-terminal truncated FGF-18 variant (FGF-18v) was shown to have improved specificity for FGF receptor-3 (FGFR-3), the major FGFR isotype involved in chondrocytes differentiation and maturation [32,33]. Correa et al. showed a clearly enhanced anabolic effect of mutated version FGF-18, signaling exclusively through FGFR-3, increasing the production and expression of ECM components (e.g. glycosaminoglycans, aggrecan, type II collagen), in comparison with wild-type FGF-18 and TGF- $\beta$ [34].

Biomechanical studies have been designed with the aim to regenerate neo-tissue resembling native healthy cartilage $[26,35,36]$. Mechanical stimulation has been shown to transcriptionally activate the expression of genes associated with various cellular processes in chondrocytes, including matrix accumulation and pro-inflammatory gene suppression [37]. Different bioreactors and loading devices have been designed to stimulate neo articular cartilage development, providing chondrocytes with optimized mechanical cues $[38,39]$. Multi-axial loading has been shown to effectively stimulate the synthesis of cartilaginous ECM macromolecules in chondrocytes cultured in 3D scaffolds [26]. Specifically, intermittent dynamic compression and sliding surface motion, applied by a ceramic ball, has been shown to improve the gene expression and the synthesis of cartilage specific matrix molecules in chondrocytes-scaffold constructs [40-44]. Both lubricin and cartilage oligomeric matrix protein gene expression are markedly enhanced by applying sliding motion to the surface of a three-dimensional scaffold, whereas the upregulation of collagen Type II and aggrecan was more associated with the application of compression [26].

Previous studies have combined mechanical loading with growth factor supplementation (e.g. fibroblast growth factor-2, transforming growth factor- $\beta$, insulin-like growth factor-1, osteogenic protein-1), to modulate chondrocytes phenotype, proliferation and biosynthetic rates [45-48]. However, to the best of our knowledge, the interplay between mechanical stimuli and FGF-18 supplementation is still unknown. We therefore investigated the effects of FGF-18v on primary chondrocytes seeded in a 3D fibrin: hyaluronan (FB/HA)-based hydrogel under free swelling and mechanical loading conditions. Cell-hydrogel constructs, in the presence or absence of FGF-18v, loaded in a custom made jointmimicking bioreactor were followed for changes in anabolic and catabolic gene expression and ECM production. This combined system may also provide an efficient, pre-clinical model for evaluating various cartilage and joint therapeutic modalities prior to animal testing and clinical translation.

\section{Materials and methods}

\subsection{Fibrin-HA hydrogel production}

The FB/HA hydrogel (3.2:1 ratio) was manufactured and provided by ProCore Biomed Inc. (Ness Ziona, Israel), at final concentrations of $6.21 \mathrm{mg} / \mathrm{mL}$ and $1.94 \mathrm{mg} / \mathrm{mL}$ of fibrinogen and HA, respectively. Fibrinogen:HA conjugates were synthesized via a twostep procedure as previously described [49]. Briefly, HA (1.55 MDa; Lifecore Biomedical, Minnesota, USA) was initially reacted with a mixture of 1-ethyl-3-(3-dimethylaminopropyl) carbodiimide (EDC; Sigma, Israel) and $N$-hydroxysuccinimide (NHS; Sigma, Israel) to convert part of its carboxylic groups to NHS-active ester moieties. In a second step, a buffered solution of fibrinogen (Omrix, Israel) was reacted with the HA active ester solution to produce a clear fibrinogen:HA conjugate solution.

\subsection{Chondrocyte isolation and culture conditions}

Chondrocytes were isolated from full thickness fetlock joint cartilage of 4-8 months old calves, using sequential pronase (Roche, Mannheim, Germany) and collagenase (Worthington Biochemical Corporation, NJ, USA) digestion [38]. Isolated chondro- 
A

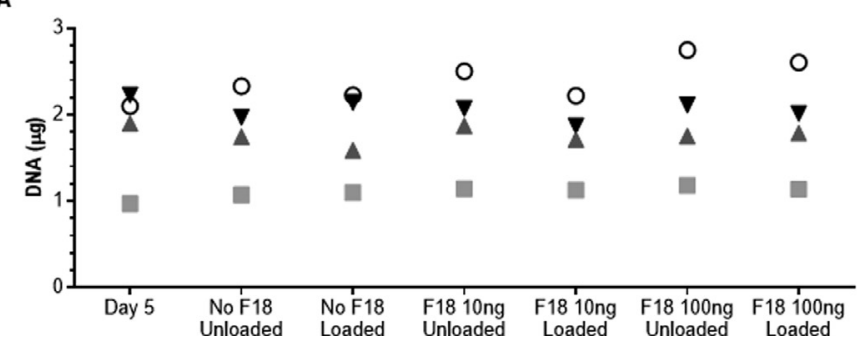

B

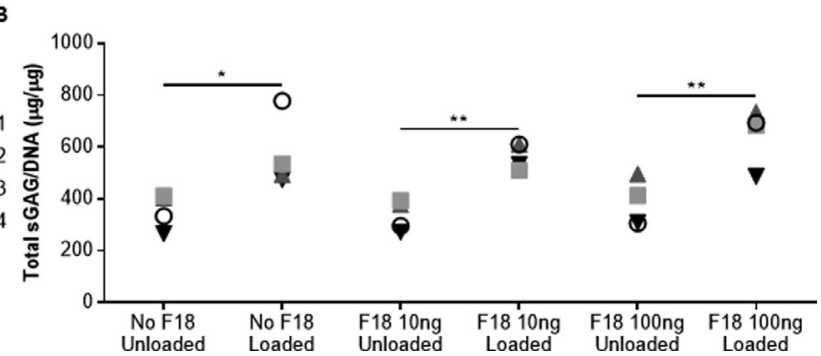

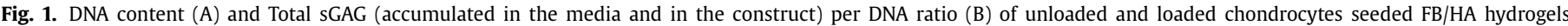

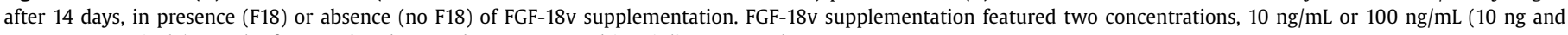
$100 \mathrm{ng}$, respectively). Results from 4 chondrocyte donors, assessed in triplicates, are shown; * $p<0.05,{ }^{* *} p<0.01$.

cytes $\left(7.5 \times 10^{6}\right.$ cells/construct) were suspended in the fibrinogen:HA conjugate $(330 \mu \mathrm{L} /$ construct) and thrombin solution (Omrix, Israel $)(22 \mu \mathrm{L} /$ construct; $50 \mathrm{U} / \mathrm{mL})$ was added at a volume ratio of 1:15 (final concentrations, FB: $5.86 \mathrm{mg} / \mathrm{mL}$; HA: $2.34 \mathrm{mg} / \mathrm{mL}$; thrombin: $3.13 \mathrm{U} / \mathrm{mL}$ ). Upon, thrombin addition, the suspension was mixed to achieve optimal cell distribution, placed in polyurethane moulds and allowed to crosslink. Cell-hydrogel constructs ( $8 \mathrm{~mm}$ diameter; $4 \mathrm{~mm}$ height) were then placed into bioreactor sample holders and incubated for $30 \mathrm{~min}$ at $37{ }^{\circ} \mathrm{C}$ and $5 \% \mathrm{CO}_{2}$, to allow complete gelation. The constructs were then cultured in growth medium (Dulbecco's Modified Eagle's medium, high glucose (DMEM-HG), 4.5 g/L-glucose; Gibco), supplemented with penicillin/streptomycin (1\% P/S, Gibco), $50 \mu \mathrm{g} / \mathrm{mL}$ ascorbic acid-2 phosphate (AA-2P, Sigma), 1\% insulin-transferrin-selenium (ITS) and non-essential amino acids. Constructs were exposed to $10 \mathrm{ng} / \mathrm{mL}$ or $100 \mathrm{ng} / \mathrm{mL}$ of FGF-18v (Procore Bio Med, Ness Ziona, Israel), added to culture media and replenished on every medium exchange. Controls not exposed to FGF-18v were included. FGF18v is a truncated version of FGF-18 lacking the amino-terminal last 50 amino acids of the ligand and has the first methionine replace glutamine 51 [32]. The medium was changed every second day, and conditioned medium was collected for analysis of sulphated glycosaminoglycans (sGAG) (Section 2.4).

\subsection{Mechanical loading}

The hydrogel-chondrocytes constructs were cultured under free swelling conditions for 5 days, to allow cell attachment, colonization and initiation of ECM deposition, similar to previously published and optimized protocol [50-52]. Subsequently, constructs were exposed to mechanical loading, in the presence or absence of different concentrations of FGF-18v. Mechanical stimuli were applied using a four-station bioreactor system, installed in an incubator at $37{ }^{\circ} \mathrm{C}, 5 \% \mathrm{CO}_{2}, 85 \%$ humidity. At each station, a commercially available ceramic hip ball (32 $\mathrm{mm}$ in diameter) was pressed onto a cell-seeded hydrogel to provide a constant displacement of $0.4 \mathrm{~mm}$ or $10 \%$ of the scaffold height (measured in the construct center). The ball oscillated vertically in a sinusoidal manner between $0.4 \mathrm{~mm}$ and $0.45 \mathrm{~mm}$, i.e., between $10 \%$ and $11.25 \%$ of the construct height, at a frequency of $0.5 \mathrm{~Hz}$. In addition to the cyclic compressive loading, reciprocate rotation of the ball about an axis perpendicular to the construct axis was promoted, at an amplitude of $25^{\circ}$ and a frequency of $0.5 \mathrm{~Hz}$ (Fig. 1). This regime of dynamic axial compression with superimposed sliding motion simulates joint articulation more closely compared to axial compression alone [38].

One hour of mechanical loading was performed daily for 14 days. In between loading cycles, the constructs were kept in a free swelling condition (without ball contact). Construct analysis was performed after a total culture time of 19 days. Unloaded scaffolds served as controls.

\subsection{Biochemical assays}

Cell-loaded hydrogels were digested overnight with $0.5 \mathrm{mg} / \mathrm{mL}$ of proteinase $\mathrm{K}$, at $56{ }^{\circ} \mathrm{C}(2.5 \mathrm{U} / \mathrm{mg}$, chromozyme assay; Roche, Mannheim, Germany). The PicoGreen ${ }^{\circledR}$ Assay (Molecular Probes, Life Technologies) was used to assess the DNA content as per manufacturer's guidelines. The sample fluorescence was measured using a microplate reader (VICTOR3 V' Multilabel Counter, PerkinElmer BioSignal Inc, USA) at $480 \mathrm{~nm}$ excitation and $520 \mathrm{~nm}$ emission. The amount of sulphated glycosaminoglycans (sGAG) was determined by a dimethylmethylene blue dye assay using DMMB solution at $\mathrm{pH} 1.5$ and bovine chondroitin sulfate as a standard. Total sGAG content of the culture media was also measured to assess the release of matrix molecules from the constructs into the media.

\subsection{Gene expression analysis}

Total RNA was extracted from homogenized constructs using TRI Reagent (Molecular Research center, Cincinnati, OH). Reverse transcription was performed with TaqMan $^{\mathrm{TM}}$ reverse transcription reagents (Thermo Fisher Scientific, Reinach, Switzerland), using random hexamer primers and $500 \mathrm{ng}$ of total RNA. PCR was performed using a QuantStudio ${ }^{\mathrm{TM}} 6$ real-time PCR instrument (Applied Biosystems) and TaqMan ${ }^{\mathrm{TM}}$ Gene Expression Master Mix. Table 1 shows the sequences of bovine primers and TaqMan $^{\mathrm{TM}}$ probes for aggrecan (ACAN), collagen type-I (COL1), type-II (COL2), type-X (COL10), cartilage oligomeric matrix protein (COMP), proteoglycan 4 (PRG4/Lubricin), matrix metalloproteinases $-3,-9$ and -13 (MMP-3, -9 and -13 ). Primers and probe for amplification of $60 \mathrm{~S}$ acidic ribosomal protein lateral stalk P0 (RPLP0, Bt03218086_m1) were acquired from Applied Biosystems (Rotkreutz, Switzerland). Relative quantification of target mRNA was performed according to the comparative CT method, using RPLP0 as an endogenous control [53].

\subsection{Histology}

Histological samples were fixed in $4 \%$ buffered formaldehyde (Formafix AG, Hittnau, CH) for $24 \mathrm{~h}$, embedded in paraffin and sectioned in $5 \mu \mathrm{m}$ sections. For staining, slides were deparaffinized using xylene and subsequently hydrated. Safranin-O/Fast green staining was performed to visualize proteoglycan and collagen deposition. Briefly, slides were first stained with Weigert's haematoxylin for $10 \mathrm{~min}$, blued in tap water for $10 \mathrm{~min}$, stained with $0.002 \%$ Fast green in deionized water for $5 \mathrm{~min}$ and washed in 1\% acetic 
Table 1

Oligonucleotide primers and probes used for qRT-PCR.

\begin{tabular}{llll}
\hline Gene & Primer forward $\left(5^{\prime}-3^{\prime}\right)$ & Primer reverse $\left(5^{\prime}-3^{\prime}\right)$ & Probe $\left(5^{\prime}\right.$ FAM-3'TAMRA $)$ \\
\hline ACAN & CCA ACG AAA CCT ATG ACG TGT ACT & GCA CTC GTT GGC TGC CTC & ATG TTG CAT AGA CCT CGC CCT CCA T \\
CoL1 & TGC AGT AAC TTC GTG CCT AGC A & CGC GTG GTC CTC TAT CTC CA & CAT GCC AAT CCT TAC AAG AGG CAA CTG C \\
COL2 & AAG AAA CAC ATC TGG TTT GGA GAA A & TGG GAG CCA GGT TGT CAT C & CAA CGG TGG CTT CCA CTT CAG CTA TGG \\
COL10 & ACT TCT CTT ACC ACA TAC ACG TGA AAG & CCA GGT AGC CCT TGA TGT ACT CA & TGC CGT TCT TAT ACA GAC CTA CCC AAG CAT G \\
CoMP & CCA GAA CGA CCA GAA & TCT GAT CTG AGT TGG GCA CCT T & ACG GCG ACC GGA TCC GCA A \\
PRG4 & GAG CAG ACC TGA ATC CGT GTA TT & GGT GGG TTC CTG TTT GTA AGT GTA & CTG AAC GCT GCC ACC TCT CTT GAA A \\
MMP-3 & GGC TGC AAG GGA CAA GGA A & CAA ACT GTT TCG TAT CCT TTG CAA & CAC CAT GGA GCT TGT TCA GCA ATA TCT AGA AAA C \\
MMP-9 & ACG AAC CAA CCT CAC CAA CAG & TGC CCC AGG AGT GTA GCC & CAG CTG GCA GAG GAA TAC CTG TAC CGC \\
MMP-13 & CCA TCT ACA CCT ACA CTG GCA AAA G & GTC TGG CGT TTT GGG ATG TT & TCT CTC TAT GGT CCA GGA GAT GAA GAC CCC \\
\hline
\end{tabular}

acid. Sections were then stained with $0.1 \%$ Safranin-0 for $12 \mathrm{~min}$ and then imaged (Zeiss Axiovert $200 \mathrm{M}$, Switzerland). Image software (National Institutes of Health, Bethesda, MD) was used for automated quantification of the intensity of red-stained sections, by colour thresholding the regions of interest and calculating the percentage of stained area.

\subsection{Immunohistochemistry}

For immunohistochemical analysis, samples were fixed in $4 \%$ buffered formaldehyde (Formafix AG, Hittnau, CH) for $24 \mathrm{~h}$, embedded in paraffin and sectioned in $5 \mu \mathrm{m}$ sections. Before immunolabeling for the aggrecan protein could be conducted, reduction and alkylation steps were necessary to expose a neoepitope. The endogenous peroxidase activity was blocked with $0.3 \%$ peroxidase in $100 \%$ methanol and the sections were enzymatically pre-treated ( $0.25 \mathrm{U} / \mathrm{ml}$ of Chondroitinase $\mathrm{ABC}$ and $25 \mathrm{mg} / \mathrm{mL}$ of hyaluronidase; both Sigma, St.Louis, MO). Next, sections were blocked with horse serum (1:20 in PBS-T) and, subsequently, incubated with the primary antibodies (overnight at $4{ }^{\circ} \mathrm{C}$ ) against Aggrecan (12/21/1-C-6, $4 \mu \mathrm{g} / \mathrm{ml}$ ) and COL2 (CIICI, $2 \mu \mathrm{g} / \mathrm{ml} \mathrm{IgG}$ ) (both Developmental Studies Hybridoma bank, University of Iowa, Iowa City, IA). The Vectastain elite $A B C$ kit mouse IgG and the ImmPACT DAB peroxidase substrate were used as detection system (both Vector Laboratories, Burlingame, CA). The cell nuclei were stained with Mayer's haematoxylin.

\subsection{Statistical analysis}

The results are expressed as mean + \pm standard deviation (SD) of four independent experiments using four chondrocyte donors $(n=12)$. As sGAG, DNA content and qPCR data did not follow normal distribution when analysed using the Shapiro-Wilk test, statistical analysis using, non-parametric, Kruskal-Wallis analysis was performed, followed by a post-hoc Dunn's comparison test. Differences were considered statistically significant for $p<0.05$.

The COL2/COL1 ratio was calculated as $2^{\wedge}(-\Delta \mathrm{Ct}(\mathrm{COL} 2)) / 2^{\wedge}(-$ $\Delta \mathrm{Ct}(\mathrm{COL} 1))$.

\section{Results}

\subsection{Mechanical stimulation promotes $S G A G$ production}

To test the biological response of chondrocytes seeded in hydrogels to mechanical loading and FGF-18v stimulation, DNA and sGAG content were quantified after 19 days in culture. All samples, independent of the application of mechanical loading and/or FGF$18 \mathrm{v}$ showed similar DNA content when compared to control samples (Day 5 - before loading; Fig. 1A). Sample groups exposed to mechanical loading produced significantly more sGAG (normalized to DNA content) when compared to unloaded samples, both in the presence or absence of FGF-18v ( $p<0.01$ for no F18 and F18 $10 \mathrm{ng}$ groups; $p<0.001$ for F18 $100 \mathrm{ng}$ group) (Fig. 1B). The sole exposure of the constructs to FGF-18v did not significantly affect sGAG production at either concentration in comparison to the samples without F18v.

To further assess the effect of the treatments on sGAG production, Safranin-O/Fast Green staining was performed (Fig. 2) and quantified (Fig. S3). Mechanical stimulation led to increased GAG deposition, when compared to unloaded controls (with and without FGF-18v supplementation). This finding is in line with sGAG/DNA results. In addition, FGF-18v supplementation did not increase proteoglycan deposition, as previously seen in the DMMB assay.

Furthermore, ACAN immunohistochemistry staining was assessed (Fig. 3). Results were found in line with those of sGAG/DNA and Safranin-O/Fast Green, displaying increased ACAN production and deposition, when exposed to mechanical loading. Such was more evident when combined with $100 \mathrm{ng} / \mathrm{mL}$ FGF-18v and without FGF-18v supplementation.

\subsection{Synergistic effect of mechanical loading and FGF-18v supplementation on cartilage gene expression}

To assess the effects of FGF-18v and the applied stimuli on the phenotype of primary chondrocytes embedded in FB/HA hydrogels, mRNA expression was evaluated after loading. Gene expression of ACAN, COMP and PRG4 was increased under loading (Fig. 4) in an FGF-18v dependent manner. Thus, the combination of mechanical loading and low FGF-18v concentration $(10 \mathrm{ng} / \mathrm{mL})$ significantly upregulated ACAN expression (Fig. 4A), when compared to loaded samples without FGF-18v (no F18 loaded; $p<0.001$ ). Moreover, both FGF-18v concentrations, in combination with mechanical loading, significantly upregulated COMP expression (Fig. 4B). FGF18v at $10 \mathrm{ng} / \mathrm{mL}$, in mechanically loaded samples, showed a significant effect when compared to unloaded samples without FGF-18 (no F18 unloaded; $p<0.01$ ). FGF18v at $100 \mathrm{ng} / \mathrm{mL}$ with mechanical loading showed a significant COMP upregulation over unloaded samples without FGF-18v (no F18 unloaded; $p<0.001$ ), unloaded samples with $10 \mathrm{ng} / \mathrm{mL}$ FGF-18v (F18 $10 \mathrm{ng}$ unloaded; $p<0.05$ ) and unloaded samples with $100 \mathrm{ng} / \mathrm{mL}$ FGF-18v (F18 $100 \mathrm{ng}$ unloaded; $p<0.0001$ ). PRG4 upregulation under load was noticeable at high FGF-18v concentration (100 ng/mL, Fig. 4C), in comparison to no F18 unloaded and unloaded samples exposed to $100 \mathrm{ng} / \mathrm{mL}$ FGF-18v (F18 $100 \mathrm{ng}$ unloaded). The comparisons found no statistical significance.

Mechanical stimulation and $100 \mathrm{ng} / \mathrm{mL}$ FGF-18v supplementation significantly upregulated COL2 expression over no F18 loaded $(p<0.05$; Fig. $5 B)$. On the other hand, treatments did not exert significant effects on COL1 and 10 expression (Fig. 5A and C, respectively). When evaluating expression levels normalized to the reference gene (absolute expression; $-\Delta \mathrm{Ct}$ ), the expression of COL2 was always higher than that of COL1 (Fig. S1A), for all time points and sample groups, which indicates a COL2/COL1 ratio favorable to 
Pre-treatments (Day 5)

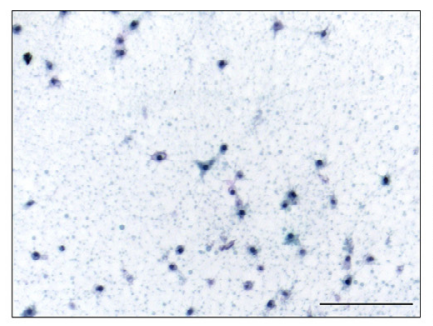

FGF-18v $10 \mathrm{ng} / \mathrm{mL}$
No FGF-18v

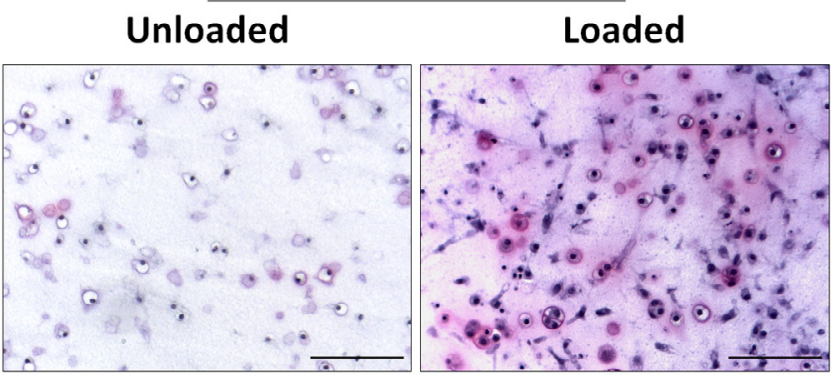

FGF-18v $100 \mathrm{ng} / \mathrm{mL}$

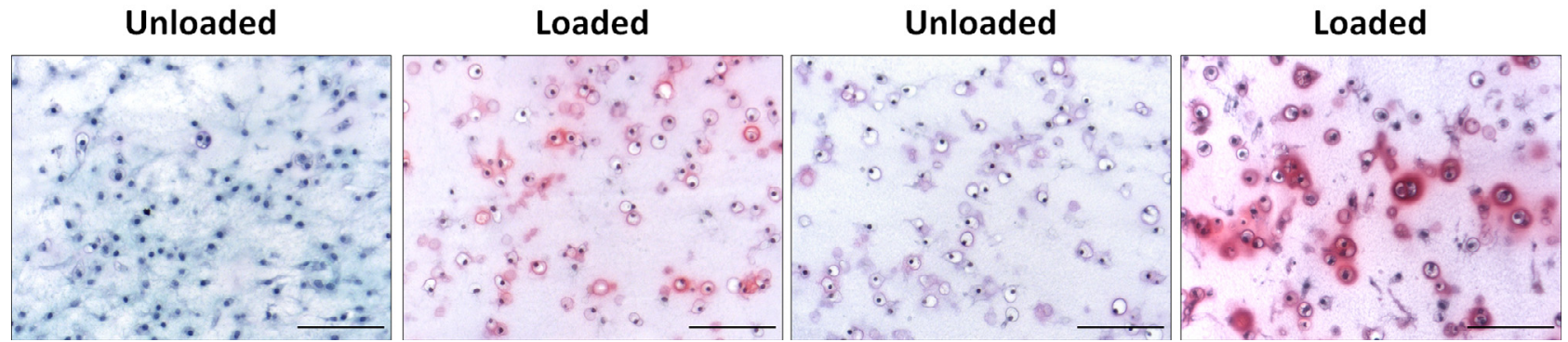

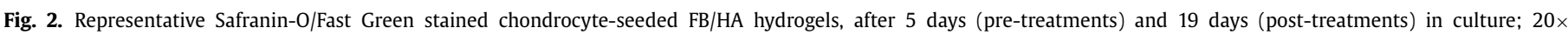
magnification, scale bars indicate $100 \mu \mathrm{m}$.

Pre-treatments

(Day 5)

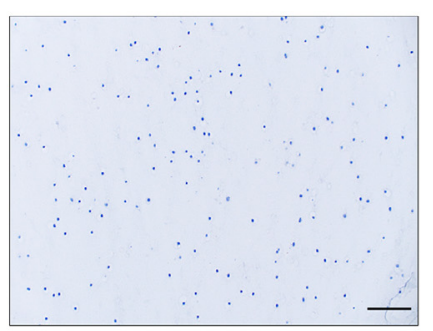

FGF-18v $10 \mathrm{ng} / \mathrm{mL}$
No FGF-18v

Unloaded Loaded
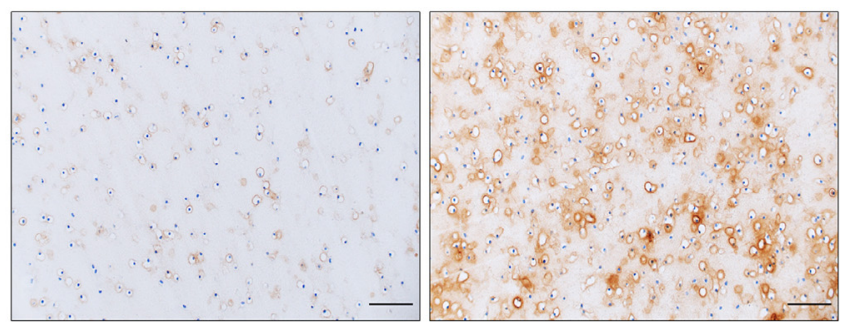

FGF-18v $100 \mathrm{ng} / \mathrm{mL}$

Unloaded

Loaded

Unloaded

Loaded
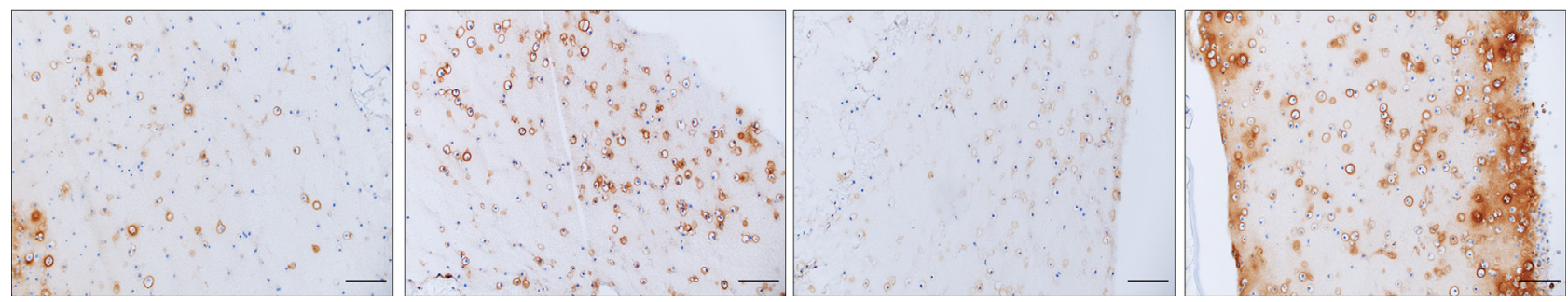

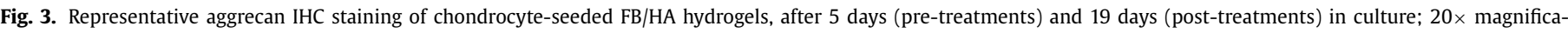
tion, scale bars indicate $100 \mu \mathrm{m}$.

COL2. To support this, COL2/COL1 ratio was calculated (Fig. S1B). Moreover, the absolute expression of COL10 was seen to drop after seeding in the hydrogel scaffolds (Day 5 - untreated control), further decreasing through time, at the end of the experiment. To complement COL2 expression, IHC staining was performed (Fig. 6). In line with gene expression findings, IHC staining revealed in- creased production and deposition of COL2 in loaded samples supplemented with $100 \mathrm{ng} / \mathrm{mL}$ FGF-18v.

When analysing MMP-9 and MMP-13 expression (Fig. 7B and C), loading significantly decreased the expression of both genes, in the presence and absence of FGF-18v. Mechanical loading, by itself, and in combination with $100 \mathrm{ng} / \mathrm{mL}$ FGF-18v was able to 
ACAN

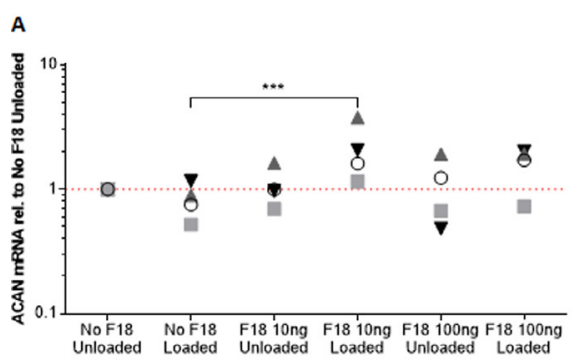

COMP

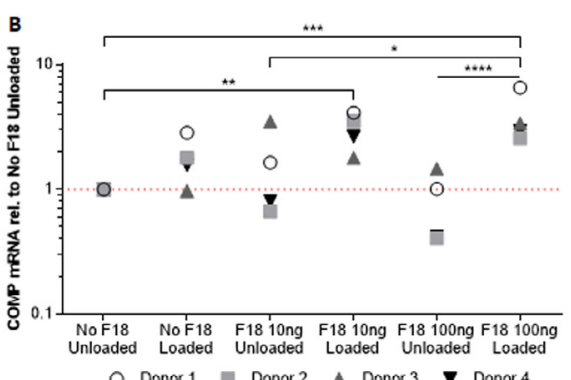

PRG4

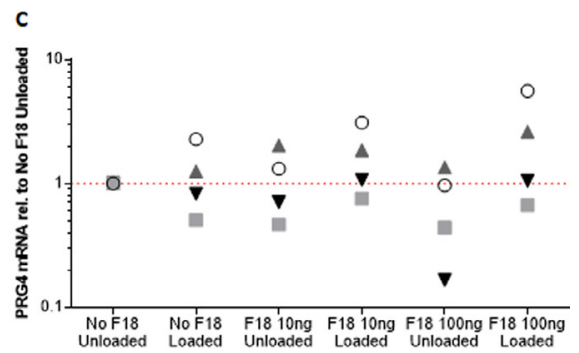

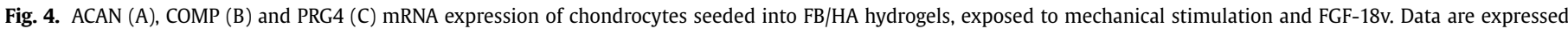

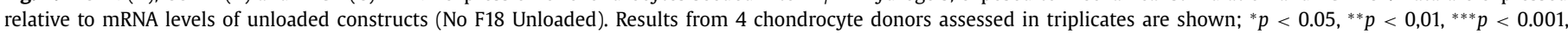
${ }^{* * * *} p<0,0001$
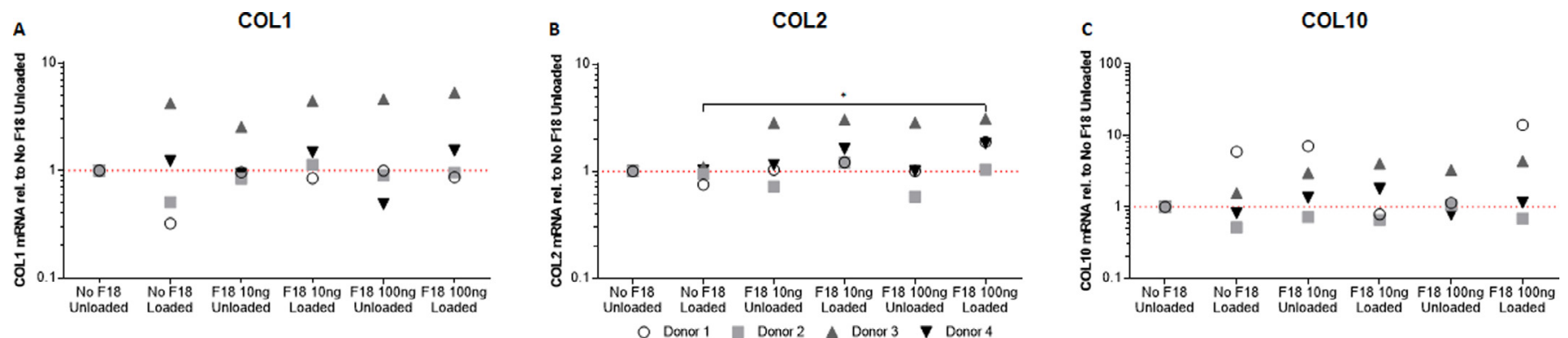

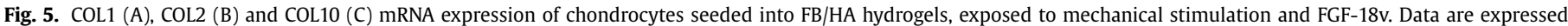
relative to mRNA levels of unloaded constructs (No F18 Unloaded). Results from 4 chondrocyte donors assessed in triplicates are shown; ${ }^{*} p<0.05$.

\section{Pre-treatments} (Day 5)

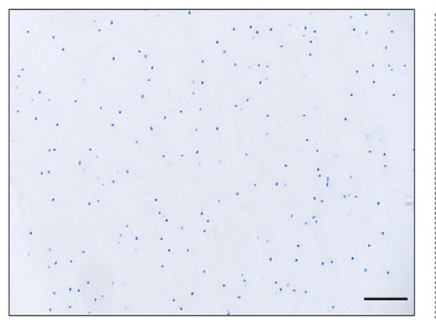

FGF-18v $10 \mathrm{ng} / \mathrm{mL}$
No FGF-18v

Unloaded

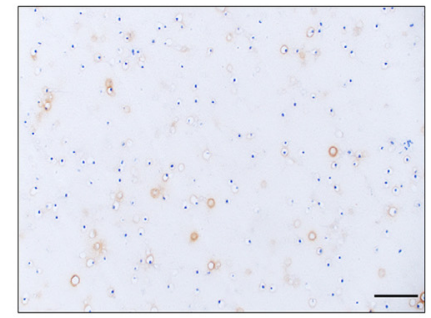

FGF-18v $100 \mathrm{ng} / \mathrm{mL}$

Loaded

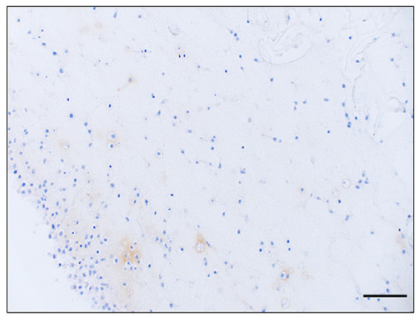

Unloaded

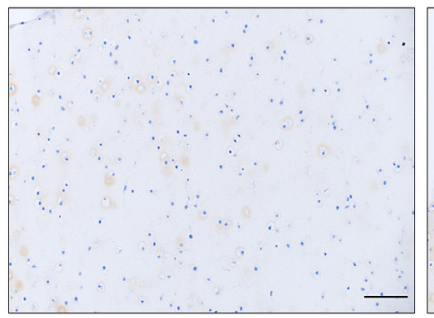

Loaded

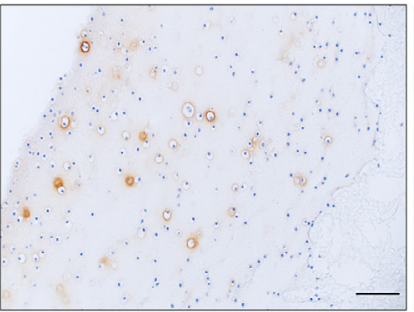

Unloaded

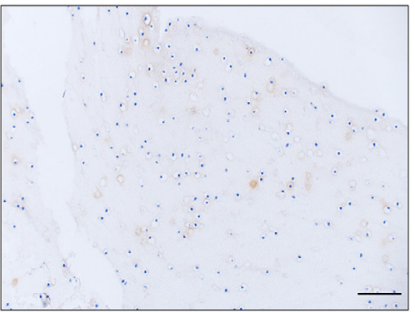

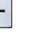

(1) 

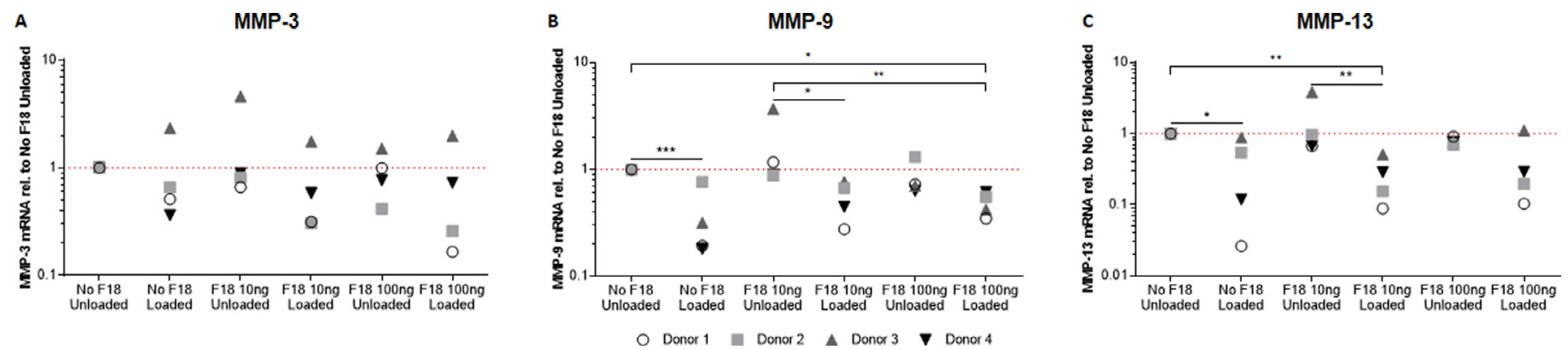

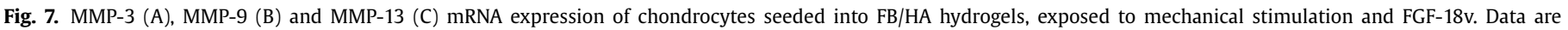

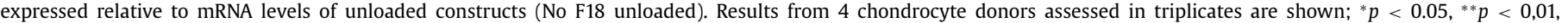
${ }^{* * *} p<0.001$.

profile seen in MMP-9 and -13 , no significant differences were found as an outcome of the treatments (Fig. 7A).

\section{Discussion}

The rationale driving this study was to create a controlled mechanochemical environment in vitro and investigate a potential synergistic effect between bi-axial mechanical stimulation and FGF-18v supplementation on primary bovine chondrocytes embedded in a FB/HA hydrogel-based 3D platform. The effect of the combined treatments on the expression of cartilage genes, matrix production and phenotype of primary bovine chondrocytes was explored in comparison to untreated controls. The combination of mechanical stimulation together with FGF-18v supplementation resulted in the upregulation of ACAN, COMP, COL2 and PRG4, while down-regulating MMPs expression. In addition, histological analysis showed increased ACAN, COL2 and GAG deposition. To the best of our knowledge, the interaction of biomimetic mechanical and FGF-18v biochemical stimuli has not been tested before, providing experimental evidence for the benefits of FGF-18v application in cartilage repair, in combination with mechanical loading.

Complex mechanical motion plays a crucial role in the development of cartilage and maintenance of the chondrogenic phenotype $[26,38,44]$. The loading protocol we used originated from protocols previously described by Grad et al. [38]. The original protocol featured the mechanical stimulation of chondrocyte-seeded polyurethane (PU) scaffolds, by applying a cyclical regime consisting of dynamic compression with superimposed sliding motion (shear). Being mechanically stiffer, PU scaffolds were subject to higher intensity set-ups, with a constant compression displacement of $10 \%$ of the scaffold's height (and a dynamic oscillation between $10 \%$ and $20 \%$ ), together with $\pm 25^{\circ}$ perpendicular shear movement, both at the frequency of $1 \mathrm{~Hz}$. Fibrin-based hydrogels used here feature lower resilience than PU scaffolds. We therefore tuned down the mechanical loading set-up to fit the mechanical profile of our constructs. Additionally, applying controlled, submaximal mechanical stimulation may have revealed a differential response of cell differentiation markers to mechanical stimulation as well as uncovered the mechanical requirements for preconditioning cells to respond to biochemical signals such that of FGF18. Moderate multi-axial loading may better represent the limited, partial weight bearing loads exerted on articular joints of patients suffering from $\mathrm{OA}$ and undergoing various treatment protocols, including intraarticular injection of the presented Fibrin-HA hydrogel (Regenogel) [54-57]. Despite using a lower intensity set-up, it is worth noting that the mechanical loading resulted in a significant increase in total sGAG production, further supported by SafraninO/Fast Green and ACAN IHC staining, when compared to unloaded controls, as previously seen for the higher intensity set-up [38]. Hence, using $\mathrm{FB} / \mathrm{HA}$ as a $3 \mathrm{D}$ platform, a similar increase in total
sGAG production was observed compared to previous studies with PU scaffolds, validating this hydrogel as a proper environment for mechanical stimulation of chondrocytes [38,44].

The provision of FGF-18v did not result in any significant changes in total sGAG production, with or without mechanical loading. Gigout et al. showed that porcine chondrocytes, in 3D pellet culture, when exposed to recombinant FGF-18 in noncontinuous fashions (one-week exposure, once/week exposure) for 5 weeks, resulted in higher matrix deposition compared to the continuously exposed ones [27]. This is called a "hit and run" effect, where short exposure periods tend to more effectively initiate a cascade response, inducing an anabolic effect. Intermittent provision of FGF-18v (once/week) was tested in the present system, for Donor 1, failing to increase sGAG production (in combination with or without mechanical loading), and therefore was dropped for the remaining donors (Fig. S2). The fact that our experiment ran for 3 weeks against the 5-week span featured in Gigout et al., may have played a role in the differences observed. Furthermore, the cell type used, the age and health of the donor should also be considered relevant to the outcome of the study.

Mechanical loading and FGF-18 supplementation have individually shown promise, in several in vitro studies, in maintaining chondrogenic phenotype and enhancing matrix production $[27,30,34,38,44,58]$. Previous studies have shown that compression was associated with an upregulation of ACAN, whereas COMP and PRG4 gene expression were markedly enhanced by shear motion at the surface of cell-seeded constructs [26]. Nonetheless, in our study mechanical stimulation by itself was not able to significantly upregulate these genes compared to unloaded samples, most likely due to the combination of lower dynamic compression applied and the low-frequency shear modulus $(0.5 \mathrm{~Hz}$ instead of $1 \mathrm{~Hz})$. Despite the provision of FGF-18v was also not sufficient to promote upregulation of these genes in unloaded samples, it is noteworthy that the combination of the factor and mechanical stimuli markedly increased the expression of cartilage matrix genes in a synergistical way (i.e. ACAN, COMP, COL2 and PRG4). Huang et al. described the upregulation of ACAN, COMP and PRG4 by FGF-18 on human adipose-derived stem cells, suggesting that a higher concentration of $100 \mathrm{ng} / \mathrm{mL}$ was more effective than a lower one $(10 \mathrm{ng} / \mathrm{mL})$ [59]. Although working on a different set of cells (i.e. primary bovine chondrocytes), by combining FGF-18v supplementation with mechanical loading we were able to achieve upregulation of ACAN and COMP on lower FGF-18v concentration (10 ng/mL). Conversely, and despite no statistical significance found, PRG4 showed increased upregulation at the highest FGF-18v concentration used. Furthermore, Correa et al. described a study, where human mesenchymal stem cells were supplemented with TGF- $\beta$ and the same variant FGF-18 herein used [34]. In a time-frame similar to ours (21 days experiment, starting FGF-18v exposure on day 7; continuous supplementation), no ACAN upregulation was achieved, indicating 
that mechanical loading is important and necessary to induce expression of cartilage matrix genes.

While the expression of COL1 and 10 showed no significant differences between treatment groups (Fig. 5A and C, respectively), the synergistic action of mechanical loading and $100 \mathrm{ng} / \mathrm{mL}$ FGF-18v led to significant COL2 upregulation (Fig. 5B), further supported by IHC analysis (Fig. 6). Furthermore, when looking at unnormalized gene expression data (Fig. S1A), COL2 overall expression was higher than COL1 expression for all groups. Thus, we calculated COL2/COL1 absolute expression ratio (Fig. S1B), confirming a ratio favorable to COL2, suggesting that the chondrocytic phenotype was maintained within the $3 \mathrm{D}$ environment in both treated and untreated conditions. This should lead to the production of hyaline cartilaginous tissue in favor of fibrocartilage [60]. In addition, in our system COL10 expression decreased after seeding into the hydrogel (Fig. S1A), further suggesting the preservation of the differentiated state of chondrocytes, not leading to hypertrophy, characteristic of OA cartilage [61].

Mechanical stimulation is known to be critical for maintaining tissue homeostasis, being a key factor in regulating the balance between chondrocyte anabolic and catabolic processes $[26,38,42,44,62]$. Consistent with other reports [58,63,64], our findings showed a decrease in the expression of the matrix degrading enzymes, MMP-9 and -13, thereby limiting ECM degradation associated with joint pathologies. This means that our treatment did not foster collagenase-induced ECM degradation, but even downregulated the expression of matrix degrading agents. Additionally, other studies have shown that FGF-18 supplementation led to a downregulation of MMP expression (e.g. MMP-2, -3, -9 and -13), which corroborates our findings [65-67]. Mori and associates suggested that such inhibitory effects are indirect, via the induction of tissue inhibitor of metalloproteinases (TIMPs), that execute anticatabolic actions [67]. These endogenous inhibitors are paramount in the regulation of the MMP activity, creating a balance between the production of active enzymes and their inhibition, thus regulating ECM turnover, tissue remodeling and cellular behavior [68]. The study performed by Mori et al., showed a decrease in MMP-9 and -13 expression by exposing articular chondrocytes to high concentration FGF-18, for a short period of time, while increasing, significantly, TIMP-1 expression [67]. While the present study ran for a longer span of time, lower dosage FGF-18v combined with mechanical loading, significantly down-regulated MMP-9 and -13 expression. This further confirm the feasibility of the 3D FB/HA platform as responsive system under load.

Biomechanical data demonstrating the superior mechanical properties of these FB/HA hydrogels, in comparison to Fibrin alone, have been previously described, including long term stability of gels containing cells in vitro, frequency-dependent storage moduli $\left(G^{\prime}\right)$ and the ratio between storage and loss moduli $\left(G^{\prime} / G^{\prime \prime}\right)$ overall indicating a solid-elastic character [8,22]. Moreover, as by definition, hydrogels are characterized by the water-retaining capacity of their polymeric networks [69]. These specific FB/HA hydrogels were found to retain more than $90 \%$ of their original water content due to the unique HA conjugation overcoming clot retraction, a physiologically inherent property of all Fibrin networks (unpublished data).

Moreover, degradation of biomaterial-based scaffolds heavily depends on the enzymatic milieu determined by tissue and cell type, and in particular by the action of matrix degrading enzymes, such as MMPs [70]. FB/HA hydrogels containing chondrocytes, or other differentiated cell types, maintain their overall structure for, at least, 4-5 weeks in vitro and more than 3 months in vivo (unpublished data), with no sign of degradation [ref]. Similarly, we did not observe any significant changes in mass of the constructs over time. Moreover, the presented increased production of matrix components and downregulation of matrix degrading enzymes, MMP-
9 and MMP-13, under loading, may further stabilize the hydrogels from biological degradation under the employed experimental conditions.

Having found the results of our study encouraging, nonetheless, the system faces certain limitations. The growth factor was not part of the regenerative system but was only added to the culture medium. For in vivo application of this formulation in cartilage injuries, FGF-18v would preferably be integrated within the hydrogel system rather than injected freely in the synovial fluid [71]. By integrating the growth factor within the hydrogel, a controlled delivery to the damaged area could be achieved, thus enhancing the regenerative process. It's also important to mention that the system does not fully mimic an in vivo scenario, since the mechanical loading introduced only featured compression and shear, not contemplating rotation force, which is featured in native articular motion $[26,58]$. Moreover, the work displayed in this manuscript does not account for the scenario following a trauma, in vivo, specifically the resulting synovial inflammation, and all the agents influencing this process. Additionally, the system herein featured, does not describe a confined system, as the cell-hydrogel construct is not surrounded by tissue (i.e. cartilage and/or bone). Thus, moving forward, progressing from an in vitro setting to an ex vivo, and ultimately, to an in vivo setting, would offer further insight about the potential regenerative effective of this platform of articular cartilage. The osteochondral defect model developed by Vainieri and associates could be an interesting ex vivo platform to continue studying the effects of the $\mathrm{FB} / \mathrm{HA}$ platform studied, mechanical loading and FGF-18v supplementation [44].

Furthermore, FB/HA hydrogels are highly porous matrixes, with no diffusion limit for molecules until $10 \mathrm{MDa}$ (unpublished data) in size, therefore hydrostatic pressure built-up would be virtually negligible. Nonetheless, since this is not a fully confined system, we cannot completely rule out a contribution of fluid movement around the chondrocytes, which has been shown to promote chondrogenesis, although the effect of pure hydrostatic pressure on the expression of mechano-regulated proteins, such as PRG4 or COMP, has not been shown $[72,73]$. It is also noteworthy that, the portrayed model features young chondrocytes from calf and not cells from older, diseased, tissues (e.g. osteoarthritic chondrocytes). While the present study is not a model for osteoarthritis, there is merit in translating the current work to osteoarthritic cells and investigate the re-differentiation potential of the presented platform.

\section{Conclusion}

In conclusion, our study revealed a synergism between multiaxial mechanical stimulation and biochemical signals delivered by FGF-18v in a fibrin-hyaluronan based hydrogel and their potential to enhance cartilage matrix deposition. This model may be most valuable in decoding the interplay between cells, scaffolds and cartilage guiding factors, elucidating signaling pathways implicated in cartilage homeostasis and repair. There may be merit in the clinical application of a hydrogel-based platform combined with a selective FGF-18 variant, particularly when combined with moderate partial weight bearing rehabilitation protocols. In light to the inherent advantages of each of the different applied stimuli, the fact that this platform can be injected, and crosslinked in situ, in an outpatient minimally invasive procedure, makes it an attractive, affordable and easily translatable platform for clinical application.

\section{Declaration of Competing Interest}

A.Y. is an employee of Procore Ltd. B.A., M.V., M.A., E.M. and S.G. declare no conflict of interest. 


\section{Funding source}

This project has received funding from the European Union's Horizon 2020 research and innovation programme under Marie Sklodowska-Curie Grant Agreement No. 642414.

\section{Supplementary material}

Supplementary material associated with this article can be found, in the online version, at doi:10.1016/j.actbio.2020.01.032.

\section{References}

[1] D. Nesic, R. Whiteside, M. Brittberg, D. Wendt, I. Martin, P. Mainil-Varlet, Cartilage tissue engineering for degenerative joint disease, Adv. Drug Deliv. Rev. 58 (2) (2006) 300-322.

[2] F. Berenbaum, Osteoarthritis as an inflammatory disease (osteoarthritis is not osteoarthrosis!), Osteoarthr. Cartil. 21 (1) (2013) 16-21.

[3] E.B. Hunziker, K. Lippuner, M.J. Keel, N. Shintani, An educational review of cartilage repair: precepts \& practice-myths \& misconceptions-progress \& prospects, Osteoarthr. Cartil. 23 (3) (2015) 334-350.

[4] C. Vinatier, J. Guicheux, Cartilage tissue engineering: from biomaterials and stem cells to osteoarthritis treatments, Ann. Phys. Rehabil. Med. 59 (3) (2016) 139-144.

[5] M. Jacobi, V. Villa, R.A. Magnussen, P. Neyret, MACI - a new era? Sports Med. Arthrosc. Rehabil. Ther. Technol. 3 (1) (2011) 10

[6] F.-Y. Fan, C.-C. Chiu, C.-L. Tseng, H.-S. Lee, Y.-N. Pan, K.-C. Yang, Glycosaminoglycan/chitosan Hydrogel for Matrix-Associated Autologous Chondrocyte Implantation: An in Vitro Study, 34 (2014) 211.

[7] H.V. Almeida, R. Eswaramoorthy, G.M. Cunniffe, C.T. Buckley, F.J. O’Brien, D.J. Kelly, Fibrin hydrogels functionalized with cartilage extracellular matrix and incorporating freshly isolated stromal cells as an injectable for cartilage regeneration, Acta Biomater. 36 (2016) 55-62.

[8] Z. Li, K.M. Kaplan, A. Wertzel, M. Peroglio, B. Amit, M. Alini, S. Grad, A. Yayon, Biomimetic fibrin-hyaluronan hydrogels for nucleus pulposus regeneration, Regen. Med. 9 (3) (2014) 309-326.

[9] T.N. Snyder, K. Madhavan, M. Intrator, R.C. Dregalla, D. Park, A fibrin/hyaluronic acid hydrogel for the delivery of mesenchymal stem cells and potential for articular cartilage repair, J. Biol. Eng. 8 (2014) 10.

[10] S.H. Park, S.R. Park, S.I. Chung, K.S. Pai, B.H. Min, Tissue-engineered cartilage using fibrin/hyaluronan composite gel and its in vivo implantation, Artif. Organs 29 (10) (2005) 838-845.

[11] L.A. Fortier, P.J. Brofman, A.J. Nixon, H.O. Mohammed, Disparate chondrocyte metabolism in three-dimensional fibrin cultures derived from autogenous or commercially manufactured fibrinogen, Am. J. Vet. Res. 59 (4) (1998) $514-$ 520.

[12] J.J. Sidelmann, J. Gram, J. Jespersen, C. Kluft, Fibrin clot formation and lysis: basic mechanisms, Semin. Thromb. Hemost. 26 (6) (2000) 605-618.

[13] D. Eyrich, A. Gopferich, T. Blunk, Fibrin in tissue engineering, Adv. Exp. Med. Biol. 585 (2006) 379-392.

[14] C.L. Yang, H.W. Chen, T.C. Wang, Y.J. Wang, A novel fibrin gel derived from hyaluronic acid-grafted fibrinogen, Biomed. Mater. 6 (2) (2011) 025009.

[15] A. Yayon, E. Neria, S. Blumenstein, B. Stern, H. Barkai, R. Zak, Y. Yaniv, BIOCART $^{\text {TM }}$ II a novel implant for 3D reconstruction of articular cartilage, Orthop. Proc. 88B (Supp II) (2006) 344.

[16] I. Eshed, S. Trattnig, M. Sharon, R. Arbel, G. Nierenberg, E. Konen, A. Yayon, Assessment of cartilage repair after chondrocyte transplantation with a fibrin-hyaluronan matrix-correlation of morphological MRI, biochemical T2 mapping and clinical outcome, Eur. J. Radiol. 81 (6) (2012) 1216-1223.

[17] M.F. Scully, V.V. Kakkar, C.A. Goodwin, M. O’Regan, Inhibition of fibrinolytic activity by hyaluronan and its alcohol ester derivatives, Thromb. Res. 78 (3) (1995) 255-258.

[18] K.L. Spiller, S.A. Maher, A.M. Lowman, Hydrogels for the repair of articular cartilage defects, Tissue Eng. Part B Rev. 17 (4) (2011) 281-299.

[19] I.L. Kim, R.L. Mauck, J.A. Burdick, Hydrogel design for cartilage tissue engineering: a case study with hyaluronic acid, Biomaterials 32 (34) (2011) 87718782.

[20] E. Sato, T. Ando, J. Ichikawa, G. Okita, N. Sato, M. Wako, T. Ohba, S. Ochiai, T. Hagino, R. Jacobson, H. Haro, High molecular weight hyaluronic acid increases the differentiation potential of the murine chondrocytic ATDC5 cell line, J. Orthop. Res. 32 (12) (2014) 1619-1627.

[21] M. Akmal, A. Singh, A. Anand, A. Kesani, N. Aslam, A. Goodship, G. Bentley, The effects of hyaluronic acid on articular chondrocytes, J. Bone Joint Surg. 87 (8) (2005) 1143-1149.

[22] M.L. Vainieri, A. Lolli, N. Kops, D. D’Atri, D. Eglin, A. Yayon, M. Alini, S. Grad, K. Sivasubramaniyan, G. van Osch, Evaluation of biomimetic hyaluronic-based hydrogels with enhanced endogenous cell recruitment and cartilage matrix formation, Acta Biomater. 10.1016 (2019) 293-303.

[23] A. Lolli, K. Sivasubramaniyan, M.L. Vainieri, J. Oieni, N. Kops, A. Yayon, G. van Osch, Hydrogel-based delivery of antimiR-221 enhances cartilage regeneration by endogenous cells, J. Control Release 309 (2019) 220-230.

[24] J.P. Garcia, J. Stein, Y. Cai, F. Riemers, E. Wexselblatt, J. Wengel, M. Tryfonidou, A. Yayon, K.A. Howard, L.B. Creemers, Fibrin-hyaluronic acid hydrogel-based delivery of antisense oligonucleotides for ADAMTS5 inhibition in co-delivered and resident joint cells in osteoarthritis, J. Control Release 294 (2019) 247-258.

[25] J.P. Zlotnicki, A.G. Geeslin, I.R. Murray, F.A. Petrigliano, R.F. LaPrade, B.J. Mann, V. Musahl, Biologic treatments for sports injuries II think tank-current concepts, future research, and barriers to advancement, part 3: articular cartilage, Orthop. J. Sports Med. 4 (4) (2016) 2325967116642433.

[26] S. Grad, D. Eglin, M. Alini, M.J. Stoddart, Physical stimulation of chondrogenic cells in vitro: a review, Clin. Orthop. Relat. Res. 469 (10) (2011) 2764-2772.

[27] A. Gigout, H. Guehring, D. Froemel, A. Meurer, C. Ladel, D. Reker, A.C. BayJensen, M.A. Karsdal, S. Lindemann, Sprifermin (rhFGF18) enables proliferation of chondrocytes producing a hyaline cartilage matrix, Osteoarthr. Cartil. 25 (11) (2017) 1858-1867.

[28] L.S. Lohmander, S. Hellot, D. Dreher, E.F. Krantz, D.S. Kruger, A. Guermazi, F. Eckstein, Intraarticular sprifermin (recombinant human fibroblast growth factor 18) in knee osteoarthritis: a randomized, double-blind, placebo-controlled trial, Arthritis Rheumatol. 66 (7) (2014) 1820-1831.

[29] M.B. Ellman, H.S. An, P. Muddasani, H.J. Im, Biological impact of the fibroblast growth factor family on articular cartilage and intervertebral disc homeostasis, Gene 420 (1) (2008) 82-89.

[30] J.L. Ellsworth, J. Berry, T. Bukowski, J. Claus, A. Feldhaus, S. Holderman, M.S. Holdren, K.D. Lum, E.E. Moore, F. Raymond, H. Ren, P. Shea, C. Sprecher, H. Storey, D.L. Thompson, K. Waggie, L. Yao, R.J. Fernandes, D.R. Eyre, S.D. Hughes, Fibroblast growth factor-18 is a trophic factor for mature chondrocytes and their progenitors, Osteoarthr. Cartil. 10 (4) (2002) 308-320.

[31] F. Eckstein, W. Wirth, A. Guermazi, S. Maschek, A. Aydemir, Brief Report: Intraarticular sprifermin not only increases cartilage thickness, but also reduces cartilage loss: location-independent post hoc analysis using magnetic resonance imaging, Arthritis Rheumatol. 67 (11) (2015) 2916-2922.

[32] A. Yayon, E. Rom, R. Sirkis, D. Strauss-Ayali, FGF-18 Truncated Variants Having Increased Receptor Specificity and Uses Thereof (US 9,226,949 B2), in: USPTO (Ed.) Hepacore Ltd., Israel, 2016.

[33] O. Rozenblatt-Rosen, E. Mosonego-Ornan, E. Sadot, L. Madar-Shapiro, Y. Sheinin, D. Ginsberg, A. Yayon, Induction of chondrocyte growth arrest by FGF: transcriptional and cytoskeletal alterations, J. Cell Sci. 115 (Pt 3) (2002) 553-562.

[34] D. Correa, R.A. Somoza, P. Lin, S. Greenberg, E. Rom, L. Duesler, J.F. Welter A. Yayon, A.I. Caplan, Sequential exposure to fibroblast growth factors (FGF) 2 9 and 18 enhances hMSC chondrogenic differentiation, Osteoarthr. Cartil. 23 (3) (2015) 443-453.

[35] F. Guilak, D.L. Butler, S.A. Goldstein, Functional tissue engineering: the role of biomechanics in articular cartilage repair, Clin. Orthop. Relat. Res. (391 Suppl) (2001) S295-S305.

[36] E.M. Darling, K.A. Athanasiou, Biomechanical strategies for articular cartilage regeneration, Ann. Biomed. Eng. 31 (9) (2003) 1114-1124.

[37] N. Fahy, M. Alini, M.J. Stoddart, Mechanical Stimulation of Mesenchymal Stem Cells: Implications for Cartilage Tissue Engineering, 36(1) (2018) 52-63.

[38] S. Grad, M. Loparic, R. Peter, M. Stolz, U. Aebi, M. Alini, Sliding motion modulates stiffness and friction coefficient at the surface of tissue engineered cartilage, Osteoarthr. Cartil. 20 (4) (2012) 288-295.

[39] R.M. Schulz, A. Bader, Cartilage tissue engineering and bioreactor systems for the cultivation and stimulation of chondrocytes, Eur. Biophys. J. 36 (4-5) (2007) 539-568.

[40] J.N. De Croos, S.S. Dhaliwal, M.D. Grynpas, R.M. Pilliar, R.A. Kandel, Cyclic compressive mechanical stimulation induces sequential catabolic and anabolic gene changes in chondrocytes resulting in increased extracellular matrix accumulation, Matrix Biol. 25 (6) (2006) 323-331.

[41] E. Wernike, Z. Li, M. Alini, S. Grad, Effect of reduced oxygen tension and long-term mechanical stimulation on chondrocyte-polymer constructs, Cell Tissue Res. 331 (2) (2008) 473-483.

[42] M.J. Stoddart, L. Ettinger, H.J. Hauselmann, Enhanced matrix synthesis in de novo, scaffold free cartilage-like tissue subjected to compression and shear, Biotechnol. Bioeng. 95 (6) (2006) 1043-1051.

[43] M.A. Wimmer, S. Grad, T. Kaup, M. Hanni, E. Schneider, S. Gogolewski, M. Alini, Tribology approach to the engineering and study of articular cartilage, Tissue Eng. 10 (9-10) (2004) 1436-1445.

[44] M.L. Vainieri, D. Wahl, M. Alini, G. van Osch, S. Grad, Mechanically stimulated osteochondral organ culture for evaluation of biomaterials in cartilage repair studies, Acta Biomater. 81 (2018) 256-266.

[45] B.D. Elder, K.A. Athanasiou, Synergistic and additive effects of hydrostatic pres sure and growth factors on tissue formation, PLoS One 3 (6) (2008) e2341.

[46] K.J. Gooch, T. Blunk, D.L. Courter, A.L. Sieminski, P.M. Bursac, G. Vunjak-Novakovic, L.E. Freed, IGF-I and mechanical environment interact to modulate engineered cartilage development, Biochem. Biophys. Res. Commun. 286 (5) (2001) 909-915.

[47] Z. Li, L. Kupcsik, S.J. Yao, M. Alini, M.J. Stoddart, Mechanical load modulates chondrogenesis of human mesenchymal stem cells through the TGF-beta pathway, J. Cell Mol. Med. 14 (6A) (2010) 1338-1346.

[48] R.L. Mauck, S.B. Nicoll, S.L. Seyhan, G.A. Ateshian, C.T. Hung, Synergistic action of growth factors and dynamic loading for articular cartilage tissue engineering, Tissue Eng. 9 (4) (2003) 597-611.

[49] B. Amit, H. Barkay-Olami, A. Yayon, Water Soluble Reactive Derivatives of Carboxy Polysaccharides and Fibrinogen Conjugates Thereof (US 8329870 B2), in: USPTO (Ed.) Hepacore Ltd., Israel, 2012.

[50] S. Grad, S. Gogolewski, M. Alini, M.A. Wimmer, Effects of simple and complex motion patterns on gene expression of chondrocytes seeded in 3D scaffolds, Tissue Eng. 12 (11) (2006) 3171-3179. 
[51] N. Wang, S. Grad, M.J. Stoddart, P. Niemeyer, N.P. Sudkamp, J. Pestka, M. Alini, J. Chen, G.M. Salzmann, Bioreactor-induced chondrocyte maturation is dependent on cell passage and onset of loading, Cartilage 4 (2) (2013) 165176.

[52] M.A. Wimmer, M. Alini, S. Grad, The effect of sliding velocity on chondrocytes activity in 3D scaffolds, J. Biomech. 42 (4) (2009) 424-429.

[53] T.D. Schmittgen, K.J. Livak, Analyzing real-time pcr data by the comparative C(T) method, Nat. Protoc. 3 (6) (2008) 1101-1108.

[54] S. Hackel, M. Zolfaghar, J. Du, S. Hoppe, L.M. Benneker, N. Garstka, M. Peroglio, M. Alini, S. Grad, A. Yayon, Z. Li, Fibrin-hyaluronic acid hydrogel (RegenoGel) with fibroblast growth factor-18 for in vitro 3D culture of human and bovine nucleus pulposus cells, Int. J. Mol. Sci. 20 (20) (2019) 5036-5054.

[55] E. Kon, G. Filardo, M. Berruto, F. Benazzo, G. Zanon, S. Della Villa, M. Marcacci, Articular cartilage treatment in high-level male soccer players: a prospective comparative study of arthroscopic second-generation autologous chondrocyte implantation versus microfracture, Am. J. Sports Med. 39 (12) (2011) 2549-2557.

[56] J. Farr, Autologous chondrocyte implantation improves patellofemoral cartilage treatment outcomes, Clin. Orthop. Relat. Res. 463 (2007) 187-194.

[57] M. Falah, G. Nierenberg, M. Soudry, M. Hayden, G. Volpin, Treatment of articular cartilage lesions of the knee, Int. Orthop. 34 (5) (2010) 621-630.

[58] S. Grad, S. Gogolewski, M. Alini, M.A. Wimmer, Effects of simple and complex motion patterns on gene expression of chondrocytes seeded in 3D scaffolds, Tissue Eng. 12 (11) (2006) 3171-3179.

[59] L. Huang, L. Yi, C. Zhang, Y. He, L. Zhou, Y. Liu, L. Qian, S. Hou, T. Weng, Synergistic effects of FGF-18 and TGF-beta3 on the chondrogenesis of human adipose-derived mesenchymal stem cells in the pellet culture, Stem Cells Int. 2018 (2018) 7139485

[60] I. Martin, M. Jakob, D. Schafer, W. Dick, G. Spagnoli, M. Heberer, Quantitative analysis of gene expression in human articular cartilage from normal and osteoarthritic joints, Osteoarthr. Cartil. 9 (2) (2001) 112-118.

[61] P.M. van der Kraan, W.B. van den Berg, Chondrocyte hypertrophy and osteoarthritis: role in initiation and progression of cartilage degeneration? Osteoarthr. Cartil. 20 (3) (2012) 223-232.

[62] H.B. Sun, Mechanical loading, cartilage degradation, and arthritis, Ann. N. Y. Acad. Sci. 1211 (2010) 37-50.
[63] M. Ferretti, R. Gassner, Z. Wang, P. Perera, J. Deschner, G. Sowa, R.B. Salter, S. Agarwal, Biomechanical signals suppress proinflammatory responses in cartilage: early events in experimental antigen-induced arthritis, J. Immunol. 177 (12) (2006) 8757-8766.

[64] D.J. Leong, X.I. Gu, Y. Li, J.Y. Lee, D.M. Laudier, R.J. Majeska, M.B. Schaffler, L. Cardoso, H.B. Sun, Matrix metalloproteinase-3 in articular cartilage is upregulated by joint immobilization and suppressed by passive joint motion, Matrix Biol. 29 (5) (2010) 420-426.

[65] C. Shu, S.M. Smith, C.B. Little, J. Melrose, Use of FGF-2 and FGF-18 to direct bone marrow stromal stem cells to chondrogenic and osteogenic lineages, Futur. Sci. OA 2 (4) (2016) FSO142.

[66] L.J. Sandell, X. Xing, C. Franz, S. Davies, L.W. Chang, D. Patra, Exuberant expression of chemokine genes by adult human articular chondrocytes in response to IL-1beta, Osteoarthr. Cartil. 16 (12) (2008) 1560-1571

[67] Y. Mori, T. Saito, S.H. Chang, H. Kobayashi, C.H. Ladel, H. Guehring, U.I. Chung, H. Kawaguchi, Identification of fibroblast growth factor-18 as a molecule to protect adult articular cartilage by gene expression profiling, J. Biol. Chem. 289 (14) (2014) 10192-10200.

[68] K. Brew, H. Nagase, The tissue inhibitors of metalloproteinases (TIMPs): an ancient family with structural and functional diversity, Biochim. Biophys. Acta 1803 (1) (2010) 55-71.

[69] N.A. Peppas, E.W. Merrill, Crosslinked polyvinyl-alcohol) hydrogels as swollen elastic networks, J. Appl. Polym. Sci. 21 (7) (1977) 1763-1770.

[70] T.A. Ahmed, M. Griffith, M. Hincke, Characterization and inhibition of fibrin hydrogel-degrading enzymes during development of tissue engineering scaffolds, Tissue Eng. 13 (7) (2007) 1469-1477.

[71] F. Canal, C.L. Presti, FGF-18 Formulation in Alginate/Collagen Hydrogels, in: U.S Patent (Ed.) ARES TRADING S.A., Switzerland, 2017.

[72] Y.W. Tarng, M.E. Casper, J.S. Fitzsimmons, J.J. Stone, J. Bekkers, K.N. An, F.C. Su, S.W. O'Driscoll, G.G. Reinholz, Directional fluid flow enhances in vitro periosteal tissue growth and chondrogenesis on poly-epsilon-caprolactone scaffolds, J. Biomed. Mater. Res. A 95 (1) (2010) 156-163.

[73] N. Mukherjee, D.B. Saris, F.M. Schultz, L.J. Berglund, K.N. An, O.D. SW, The enhancement of periosteal chondrogenesis in organ culture by dynamic fluid pressure, J. Orthop. Res. 19 (4) (2001) 524-530. 